\title{
Quantitative Untersuchung der Anhangsorgane der Haut bei einem Bantu-M'gonie.'
}

\author{
Von \\ Toratoshi Taniguchi. \\ Aus dem Anatonischen Institut der Keio Universität, Tokyo.
}

\section{Einleitung.}

Um die Mengenverhältnisse der Hautorgane gründlich zu ermitteln, sind seit Jahren in unserem Institut ausgedehnte quantitative Untersuchungen an den verschiedenen Körperteilen des Menschen und bei verschiedenen Rassen, Altersstufen und Geschlechtern durchgeführt worden. Untersuchungen an verschiedenen Menschenrassen liegen schon vor von Yamada an Deutschen, von Kawai an Ainu, von Kawaji an Finnen, von Shibayama an Bantu-Xosa, von Taniguchi, Nakano und Kosaka sowie Shibata ${ }^{2)}$ an Koreanern.

In der vorliegenden Abhandlung teile ich die Befunde an einem afrikanischen Eingeborenen (Bantu-M'gonie) mit.

\section{Material und Methode.}

Das Material, das Herr Prof. Dart vom Anatomischen Institut zu Witwatersland Herrn Prof. K. Okajima überreicht hat, stammt von einem an Enteritis und Herzfehler gestorbenen 26 jährigen männlichen Bantu (M'gonie-Familie). Die Leiche wurde sofort nach dem Tode mit der eine geringe Menge von Karbol und Arsen enthaltenden Formalin-

1) Diese Abhandlung ist die 13. Mitteilung von "Quantitative Untersuchungen der Anhangsorgane der Haut von K. Oka jima."

2) Der Vortrag darüber wurde in der 42. Versammlung der Japan. Anat. Gesellschaft ('Tokyo, 1934) gehalten. 
lösung im ganzen Körper injiziert und fixiert. Die davon entnommenen Hautstücke wurden in 10\%ige Formaliulösung gebracht und darin konserviert. Die Hautstücke in der Grösse von ca. $1.2 \mathrm{qcm}$ wurden aus diesem Material herausgeschnitten, in Zelloidin eingebettet und in Flächenschnittserien von $40 \mu$ Dicke zerlegt.

Es wurden 7 verschiedene Kōrperteile untersucht.

1. Hinterhaupt

2. Rücken (rechte Seite)

3. Bauch (linke Seite, etwas höher als Nabel)

4. Oberarm (Streckseite der mittleren Höhe, rechts)

5. Vorderarm (Streckseite der mittleren Höhe, rechts)

6. Oberschenkel (laterale Seite der oberen Höhe, links)

7. Unterschenkel (laterale Seite der oberen Höhe, links).

Die Färbung geschah mit Hämatoxylin(Hansen)-Eosin.

Zur Berechnung brauchte ich die Gleichung nach Okajima. Die sonstigen Behandlungen nahm ich nach den anderen Autoren unseres Institutes vor.

\section{Eigene Befunde.}

Ich werde in den folgenden Tabellen (Tab. 1-4) meine Daten zeigen.

Tabelle 1.

\begin{tabular}{|c|c|c|c|c|c|c|c|c|c|c|c|}
\hline \multirow[b]{2}{*}{$\begin{array}{l}\text { Untersuchte } \\
\text { Körperteile }\end{array}$} & \multirow{2}{*}{ 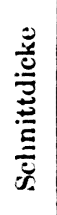 } & \multicolumn{3}{|c|}{ Schnittzahl } & \multirow{2}{*}{ 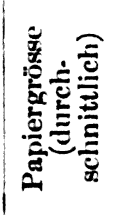 } & \multicolumn{3}{|c|}{ Papiergewicht } & \multirow{2}{*}{ 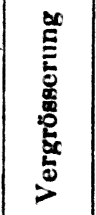 } & \multirow{2}{*}{ 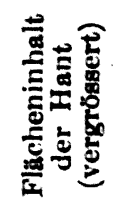 } & \multirow{2}{*}{ 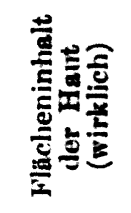 } \\
\hline & & 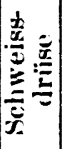 & 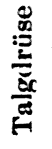 & 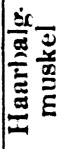 & & 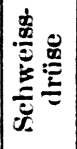 & 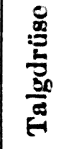 & 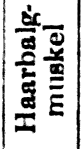 & & & \\
\hline Hinterhaupt & $40 \mu$ & 32 & 22 & 24 & $\begin{array}{c}365 \times 254 \\
\mathrm{qcm}\end{array}$ & $9.8 \mathrm{gr}$ & $9.8 \mathrm{gr}$ & $9.8 \mathrm{gr}$ & $30 \mathrm{mal}$ & $\begin{array}{c}\pi r^{2} \mathrm{gcm} \\
(\mathrm{r}=10 \mathrm{~cm})\end{array}$ & $\begin{array}{c}\pi r^{2} g \mathrm{~mm} \\
\left(r=\frac{10}{3} \mathrm{~mm}\right)\end{array}$ \\
\hline Rücken & , & 50 & 17 & 12 & " & $"$ & " & " & ” & ” & " \\
\hline Bauch & $"$ & 35 & 9 & 19 & , & , & $"$ & , & " & $"$ & ", \\
\hline Oberarm & $"$ & 27 & 11 & 17 & , & , & , & " & $"$ & $"$ & " \\
\hline Vorderarm & $"$ & $: 32$ & 9 & $\mathbf{2 3}$ & , & $"$ & $"$ & $"$ & $"$ & $"$ & " \\
\hline Oberschenkel & , & 26 & 5 & 12 & , & , & $"$ & $"$ & ", & " & $"$ \\
\hline Unterschenkel & $"$ & 25 & 3 & 12 & , & , & $"$ & $"$ & $n$ & $"$ & , \\
\hline
\end{tabular}


Quantitative Untersuchung der Anhangsorgane der Haut bei einem Bantu-M'gonie. 479

\begin{tabular}{|c|c|c|c|c|c|c|c|c|c|c|c|c|c|c|c|c|c|c|c|}
\hline & 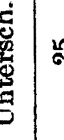 & เ్రి & జ్ఞ్ & $\begin{array}{l}\overrightarrow{18} \\
\stackrel{8}{\$ \& ~}\end{array}$ & 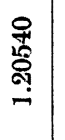 & 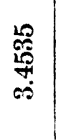 & 苞 & $\therefore$ & + & 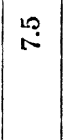 & 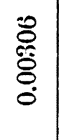 & $\begin{array}{l}\infty \\
\stackrel{0}{0} \\
\varnothing \\
0\end{array}$ & $\overrightarrow{0}$ & $\cong$ & $\overline{0}$ & $\underset{\overbrace{}}{\mathbb{N}}$ & 点 & 总 & $\underset{+}{+}$ \\
\hline & 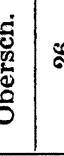 & 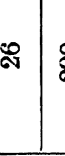 & \&્టे & $\begin{array}{l}\vec{x} \\
\text { 紟 } \\
\text { o }\end{array}$ & $\begin{array}{l}\stackrel{0}{0} \\
\text { 范 } \\
\stackrel{0}{0}\end{array}$ & 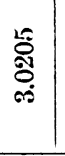 & $\stackrel{\text { ஸ̣ }}{\circ}$ & is & $\simeq$ & $\hat{\infty}$ & $\begin{array}{l}\vec{i} \\
i \\
0 \\
0 \\
0\end{array}$ & $\begin{array}{l}\text { 管 } \\
\stackrel{0}{0} \\
\dot{0}\end{array}$ & $\ddot{0}$ & 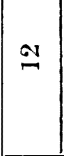 & 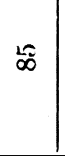 & 离 & 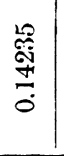 & $\begin{array}{l}\infty \\
\stackrel{\infty}{0} \\
\stackrel{+}{0} \\
0\end{array}$ & $\bar{F}$ \\
\hline & 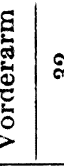 & హ్ & 峹 & $\begin{array}{l}\bar{x} \\
\stackrel{q}{q}\end{array}$ & 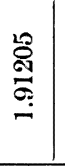 & 足 & $\begin{array}{l}\infty \\
\text { 吕 }\end{array}$ & $\sigma$ & $\stackrel{2}{2}$ & 今ે & $\begin{array}{l}\mathscr{8} \\
\text { ôे } \\
\stackrel{0}{0} \\
\dot{0}\end{array}$ & 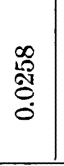 & \%ి & 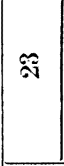 & $\stackrel{+10}{2}$ & 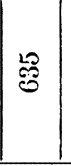 & 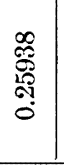 & 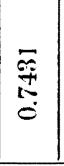 & $\stackrel{+}{\stackrel{H}{*}}$ \\
\hline & 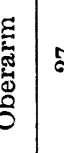 & ลิ & 芯 & $\begin{array}{l}100 \\
\stackrel{10}{\circ} \\
\stackrel{\leftrightarrow}{9}\end{array}$ & \begin{tabular}{c}
\multirow{1}{0}{} \\
0 \\
$\infty$ \\
0 \\
0
\end{tabular} & 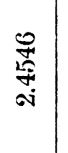 & 足 & $\Rightarrow$ & $\stackrel{N}{\Upsilon}$ & 尽 & 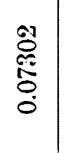 & $\begin{array}{l}\overline{\tilde{\sigma}} \\
\text { ণ़ } \\
\text { d. }\end{array}$ & $\overrightarrow{\mathrm{a}}$ & $\simeq$ & $\underset{\sim}{\stackrel{g}{2}}$ & 壱 & 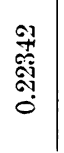 & 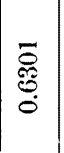 & ْి \\
\hline & 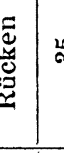 & هִ & $\stackrel{2}{\%}$ & $\begin{array}{l}\infty \\
\stackrel{0}{+} \\
\text { +1 } \\
\text { a }\end{array}$ & 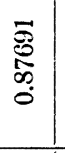 & 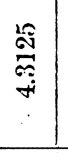 & $\overrightarrow{\tilde{F}}$ & 0 & $\widetilde{\Im}$ & $\stackrel{10}{+}$ & $\begin{array}{l}\infty \\
\stackrel{\infty}{\infty} \\
\stackrel{0}{0} \\
0\end{array}$ & 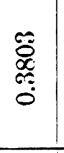 & $\stackrel{\circ}{\circ}$ & $\stackrel{\Omega}{\Omega}$ & $\stackrel{\circ}{\circ}$ & $\stackrel{\stackrel{\infty}{\infty}}{\sim}$ & 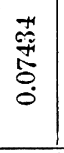 & $\stackrel{\infty}{\stackrel{0}{\circ}}$ & $\bar{z}$ \\
\hline & 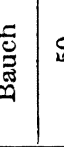 & 옹 & $\stackrel{\infty}{\infty}$ & 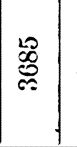 & 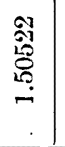 & 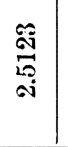 & 仿 & $\approx$ & 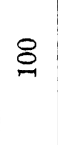 & $\stackrel{4}{6}$ & 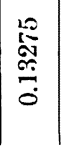 & $\begin{array}{l}\overrightarrow{\widehat{O}} \\
\text { 웅 } \\
\stackrel{0}{0}\end{array}$ & $\stackrel{10}{\circ}$ & $\stackrel{N}{工}$ & $\vec{\varepsilon}$ & $\underset{\leftrightarrow}{\stackrel{*}{*}}$ & \begin{tabular}{l}
10 \\
$\infty$ \\
$\infty$ \\
$\approx$ \\
\hdashline \\
0 \\
0
\end{tabular} & $\frac{\stackrel{O}{0}}{\stackrel{\leftrightarrow}{N}}$ & $\overrightarrow{\mathrm{i}}$ \\
\hline & 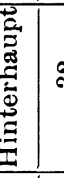 & $\stackrel{\mathcal{F}}{\propto}$ & $\underset{\infty}{20}$ & 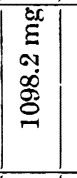 & 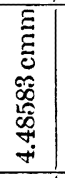 & 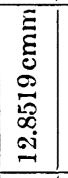 & 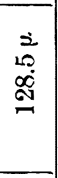 & 今̀ & 옹 & 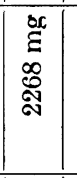 & 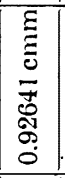 & 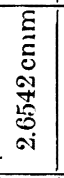 & $\begin{array}{l}2 \\
10 \\
00 \\
\infty 1\end{array}$ & $\overleftrightarrow{N}$ & $\stackrel{\infty}{20}$ & $\begin{array}{c}\infty \\
\stackrel{\infty}{\Xi} \\
10 \\
\stackrel{0}{=} \\
=\end{array}$ & 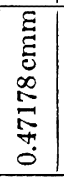 & 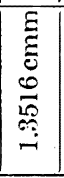 & 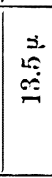 \\
\hline & 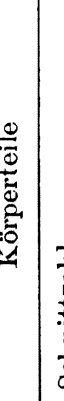 & $=$ & 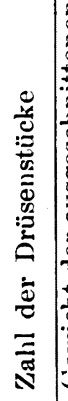 & 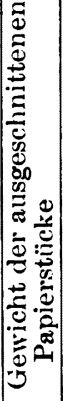 & 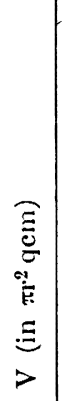 & \begin{tabular}{c}
$\widehat{E}$ \\
$\stackrel{\Xi}{0}$ \\
\hdashline \\
$\Xi$ \\
$>$
\end{tabular} & 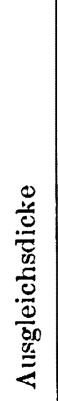 & 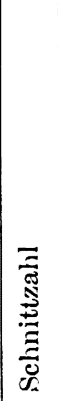 & 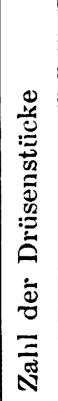 & 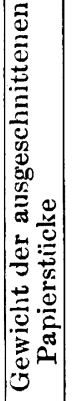 & \begin{tabular}{|c} 
\\
\\
$\widehat{\xi}$ \\
$\tilde{E}$ \\
$\sigma$ \\
$\tilde{E}$ \\
$\Xi$ \\
$\Xi$ \\
$D$
\end{tabular} & 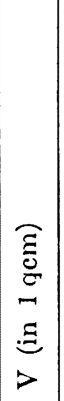 & 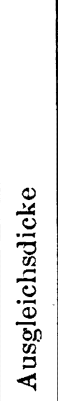 & 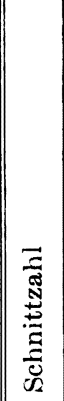 & 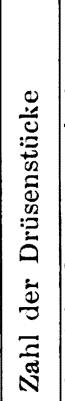 & 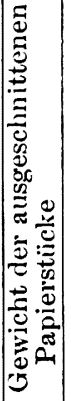 & 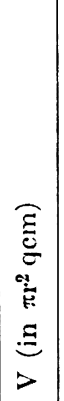 & 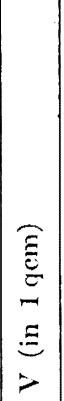 & 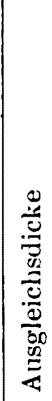 \\
\hline & & & & 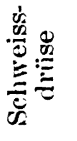 & & & & & & 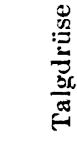 & & & & & & & & & \\
\hline
\end{tabular}


Toratoshi Taniguchi,

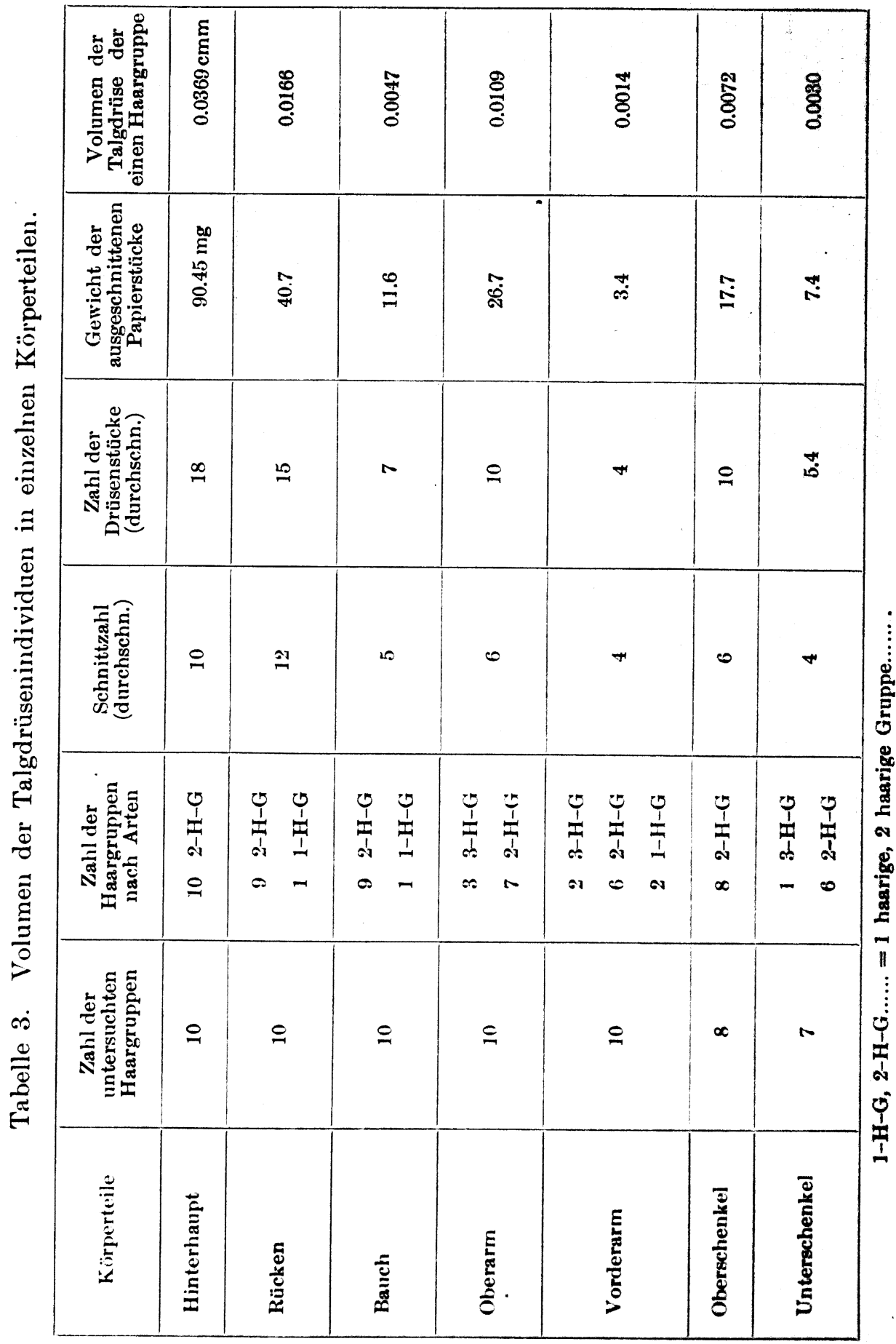


Quantitative Untersụchung der Anhangsorgane der Haut bei einem Bantu-M'gonie. 481

\begin{tabular}{|c|c|c|c|c|c|c|c|c|}
\hline 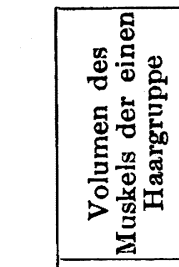 & 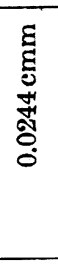 & $\begin{array}{l}\widehat{o ̛ ̊} \\
0 \\
0\end{array}$ & 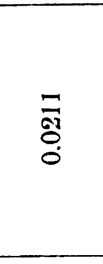 & 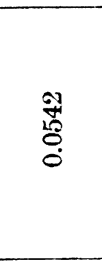 & & 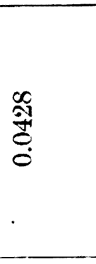 & $\begin{array}{l}\hat{0} \\
\stackrel{0}{\circ} \\
\stackrel{0}{0} \\
0 \\
0\end{array}$ & $\begin{array}{l}\infty \\
\stackrel{0}{0} \\
\stackrel{0}{0}\end{array}$ \\
\hline 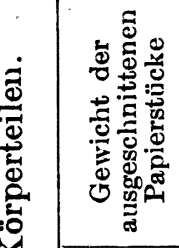 & 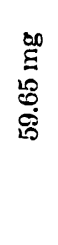 & 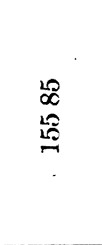 & $\frac{10}{\stackrel{10}{10}}$ & $\begin{array}{l}12 \\
\stackrel{1}{0} \\
0 \\
\stackrel{9}{0}\end{array}$ & & 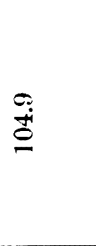 & 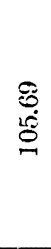 & 莣 \\
\hline | & $\stackrel{8}{*}$ & $\hat{\imath}$ & $\bar{\sigma}$ & $\bar{F}$ & & 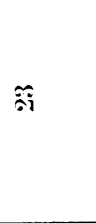 & $\stackrel{\infty}{\stackrel{N}{*}}$ & $\Leftrightarrow$ \\
\hline 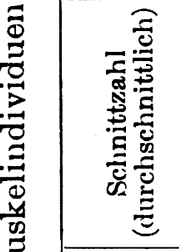 & $\stackrel{20}{2}$ & $\stackrel{\leftrightarrow}{\sim}$ & $\stackrel{10}{\sim}$ & $\stackrel{10}{=}$ & & $\approx$ & $\cong$ & 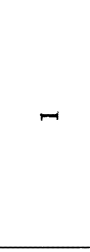 \\
\hline 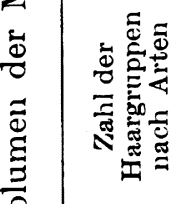 & $\begin{array}{l}5 \\
1 \\
1 \\
1 \\
1 \\
0 \\
0\end{array}$ & $\begin{array}{ll}0 & 0 \\
1 & 1 \\
1 & 1 \\
\vdots & 1 \\
\vdots & 1 \\
0 & -1\end{array}$ & 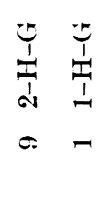 & 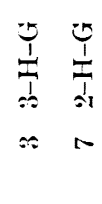 & $\begin{array}{c}1 \\
\frac{1}{T} \\
\infty \\
\infty\end{array}$ & $\begin{array}{ll}0 & 5 \\
1 & \frac{1}{1} \\
0 & 1 \\
0 & 0\end{array}$ & $\begin{array}{l}1 \\
\frac{1}{1} \\
\infty \\
\infty\end{array}$ & 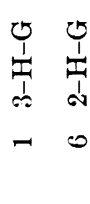 \\
\hline 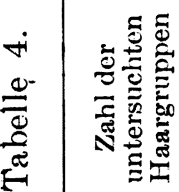 & 으 & 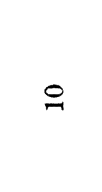 & $\stackrel{\circ}{2}$ & 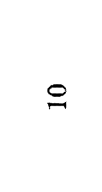 & & $\subseteq$ & $\propto$ & $\sim$ \\
\hline 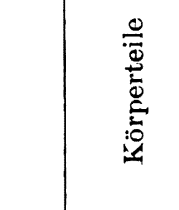 & 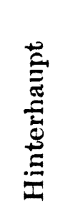 & 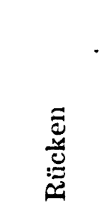 & 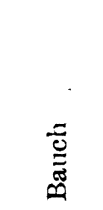 & 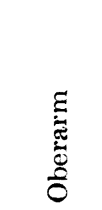 & & 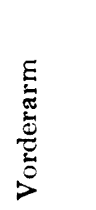 & 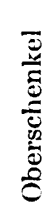 & 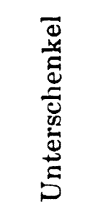 \\
\hline
\end{tabular}




\section{Tabellarische Zusammenstellung.}

Im folgenden möchte ich die obigen Befunde noch einınal tabellarisch zusammenstellen.

1. Schweissdrüse.

a. Menge und Ausmündungszahl der Drüse in den einzelnen Körperteilen.

Tabelle I. (Schweissdrüse).

\begin{tabular}{|l|c|c|c|c|}
\hline Körperteile & $\begin{array}{c}\text { Volumen der } \\
\text { Schweissdrüse } \\
\text { in 1 qcm Haut- } \\
\text { flächeninhalt }\end{array}$ & $\begin{array}{c}\text { Angenommene } \\
\text { Ausgleichsdicke } \\
\text { (rund) }\end{array}$ & $\begin{array}{c}\text { Angenommene } \\
\text { Volumen der } \\
\text { Schweissdrüsen- } \\
\text { individuen }\end{array}$ & $\begin{array}{c}\text { Ausminndungs- } \\
\text { zahl der } \\
\text { Schweissdrüse } \\
\text { in 1 qcm }\end{array}$ \\
\hline Hinterhaupt & $12.8519 \mathrm{cmm}$ & $128.5 \mu$ & $0.0345 \mathrm{cmm}$ & 373 \\
Bauch & 2.5123 & 25.1 & 0.0189 & 138 \\
Rücken & 4.3125 & 43.1 & 0.0282 & 153 \\
Oberarm & 2.4546 & 24.5 & 0.0166 & 148 \\
Vorderarm & 5.4770 & 54.8 & 0.0257 & 213 \\
Obersch. & 3.0205 & 30.2 & 0.0191 & 158 \\
Untersch. & 3.4535 & 34.5 & 0.0219 & 758 \\
\hline
\end{tabular}

b. Reihenfolge der Meugengrösse und Ausmündungszahl der Drüse in einzelnen Körperteilen.

Tabelle II. (Schweissdrüse, Reihenfolge).

\begin{tabular}{|c|c|c|c|c|c|}
\hline \multicolumn{2}{|c|}{$\begin{array}{l}\text { Volumen der Schweissdrüse } \\
\text { in } 1 \text { qcm }\end{array}$} & \multicolumn{2}{|c|}{$\begin{array}{l}\text { Volumen der angenommenen } \\
\text { Drïsenindividuen }\end{array}$} & \multicolumn{2}{|c|}{$\begin{array}{l}\text { Ausminndungszahl der } \\
\text { Schweissdrüse in } 1 \mathrm{qcm}\end{array}$} \\
\hline Körperteile & Volumen & Körperteile & Volumen & Körperteile & Zahl \\
\hline Hinterhaupt & $12.8519 \mathrm{cmm}$ & Hinterhaupt & $0.0345 \mathrm{cmm}$ & Hinterhaupt & 373 \\
\hline Vorderarm & 5.4770 & Rücken & 0.0282 & Vorderarm & 213 \\
\hline Rücken & 4.3125 & Vorderarm & 0.0257 & Obersch. & 158 \\
\hline Untersch. & 3.45335 & Untersch. & 0.0219 & Untersch. & 158 \\
\hline Obersch. & 3.0205 & Obersch. & 0.0191 & Rūcken & 153 \\
\hline Bauch & 2.5123 & Bauch & 0.0189 & Oberarm & 148 \\
\hline Oberarm & 2.4546 & Oberarm & 0.0166 & Bauch & 138 \\
\hline
\end{tabular}


Quantitative Untersuchung der Anhangsorgane der Haut bei einem Bantu-M'gonie. 483

c. Menge und Ausmündungszahl der Drüse in den Hauptkörperabschnitten.

Tabelle III. (Schweissdrüse).

\begin{tabular}{|c|c|c|c|c|c|c|}
\hline \multirow{2}{*}{ Körperteile } & \multicolumn{2}{|c|}{$\begin{array}{l}\text { Volumen der Schweiss- } \\
\text { drüse in } 1 \mathrm{gcm}\end{array}$} & \multicolumn{2}{|c|}{$\begin{array}{l}\text { Volumen der angenomme- } \\
\text { nen Drüsenindividuen }\end{array}$} & \multicolumn{2}{|c|}{$\begin{array}{l}\text { Ausmündungszahl } \\
\text { der Schweissdrüse } \\
\text { in } 1 \mathrm{qcm}\end{array}$} \\
\hline & Körperteile & Volumen & Körperteile & Volumen & Körperteile & Zahl \\
\hline \multirow{3}{*}{$\begin{array}{l}\text { ganzer } \\
\text { Körper }\end{array}$} & Kopf & $12.8519 \mathrm{cmm}$ & Kopf & $0.0345 \mathrm{cmm}$ & Kopf & 875 \\
\hline & Extremität & 3.6013 & Rumpf & 0.0236 & Extremität & 169 \\
\hline & Rumpf & 3.4124 & Fxtremität & 0.0208 & Rumpf & 146 \\
\hline \multirow{2}{*}{ Rumpf } & Rücken & 4.3125 & Rüicken & 0.0282 & Rücken & 153 \\
\hline & Bauch & 2.5123 & Bauch & 0.0189 & Bauch & 138 \\
\hline \multirow{2}{*}{$\begin{array}{l}\text { Extremität } \\
\text { (a) }\end{array}$} & obere Extr. & 3.9658 & obere Extr. & 0.0212 & obere Extr. & 181 \\
\hline & untere Fxtr. & .3 .2370 & untere Extr & 0.0205 & untere Extr & 158 \\
\hline \multirow{2}{*}{$\begin{array}{l}\text { Extremität } \\
\text { (b) }\end{array}$} & Distalis & 4.4653 & Distalis & 0.0235 & Distalis & 186 \\
\hline & Proximalis & 2.7375 & Proximalis & 0.0179 & Proximalis & 153 \\
\hline \multirow{2}{*}{$\begin{array}{l}\text { obere } \\
\text { Extremität }\end{array}$} & Vorderarm & 5.4770 & Vorderarm & 0.0257 & Vorderarm & $21: 3$ \\
\hline & Oberarm & 2.4546 & Oberarm & 0.0166 & Oberarm & 148 \\
\hline \multirow{2}{*}{$\begin{array}{l}\text { untere } \\
\text { Extremität }\end{array}$} & Untersch. & 3.4535 & Untersch. & 0.0219 & Obersch. & 158 \\
\hline & Obersch. & 3.0205 & Obersch. & 0.0191 & Untersch. & 158 \\
\hline
\end{tabular}


2. Talgdrüse.

a. Menge der Drüse in den einzelnen Körperteilen.

Tabelle IV. (Talgdrūse).

\begin{tabular}{|c|c|c|c|c|c|}
\hline \multirow[b]{2}{*}{ Körperteile } & \multirow{2}{*}{$\begin{array}{l}\text { Volumen } \\
\text { der } \\
\text { Talgdrüse } \\
\text { in } 1 \mathrm{gcm}\end{array}$} & \multirow{2}{*}{$\begin{array}{l}\text { Angenom- } \\
\text { mene } \\
\text { Ausgleichs- } \\
\text { dicke } \\
\text { (rund) }\end{array}$} & \multirow{2}{*}{$\begin{array}{l}\text { Volumen } \\
\text { der } \\
\text { Talgdrüsen- } \\
\text { individuen } \\
\text { (durchschn.) }\end{array}$} & \multicolumn{2}{|c|}{ Reihenfolge nach Volumen } \\
\hline & & & & in $1 \mathrm{qcm}$ & $\begin{array}{l}\text { Drüsenindivi- } \\
\text { duum (durch- } \\
\text { schnittlich) }\end{array}$ \\
\hline Hinterhaupt & $\begin{array}{c}\mathrm{cmm} \\
2.6542\end{array}$ & $26.5 \mu$ & $\begin{array}{c}\mathrm{cmm} \\
0.0369\end{array}$ & Hinterhaupt & Hinterhanpt \\
\hline Bauch & 0.0521 & 0.5 & 0.0047 & Rücken & Rücken \\
\hline Rücken & 0.3803 & 3.8 & 0.0166 & Oberarm & Oberarm \\
\hline Oberarm & 0.2091 & 2.1 & 0.0109 & Bauch & Obersch. \\
\hline Vorderarm & 0.0258 & 0.3 & 0.0014 & Obersch. & Bauch \\
\hline Obersch. & 0.0433 & 0.4 & 0.0072 & Vorderarm & Untersch. \\
\hline Untersch. & 0.0088 & 0.1 & 0.0030 & Untersch. & Vorderarm \\
\hline
\end{tabular}

b. Menge der Drüse in den Hauptkörperabschnitten.

Tabelle V. (Talgdrüse).

\begin{tabular}{|c|c|c|c|c|}
\hline \multirow{2}{*}{ Körperteile } & \multicolumn{2}{|c|}{$\begin{array}{l}\text { Volumen der Talgdrüse } \\
\text { in } 1 \mathrm{gcm}\end{array}$} & \multicolumn{2}{|c|}{$\begin{array}{l}\text { Volumen der Talgdrüsenindiv. } \\
\text { (durchschnittlich) }\end{array}$} \\
\hline & Körperteile & Volumen & Körperteile & Volumen \\
\hline ganzer Körper & $\begin{array}{l}\text { Kopf } \\
\text { Rumpf } \\
\text { Extremität }\end{array}$ & $\begin{array}{l}2.6542 \mathrm{cmm} \\
0.2162 \\
0.0718\end{array}$ & $\begin{array}{l}\text { Kopf } \\
\text { Rumpf } \\
\text { Extremität }\end{array}$ & $\begin{array}{l}0.0369 \mathrm{cmm} \\
0.0107 \\
0.0056\end{array}$ \\
\hline Rumpf & $\begin{array}{l}\text { Rücken } \\
\text { Bauch }\end{array}$ & $\begin{array}{l}0.3803 \\
0.0521\end{array}$ & $\begin{array}{l}\text { Rücken } \\
\text { Bauch }\end{array}$ & $\begin{array}{l}0.0166 \\
0.0047\end{array}$ \\
\hline Extremität (a) & $\begin{array}{l}\text { obere Extr. } \\
\text { untere Extr. }\end{array}$ & $\begin{array}{l}0.1175 \\
0.0261\end{array}$ & $\begin{array}{l}\text { obere Extr. } \\
\text { untere Extr. }\end{array}$ & $\begin{array}{l}0.0062 \\
0.0051\end{array}$ \\
\hline Fxtremität (b) & $\begin{array}{l}\text { Proximalis } \\
\text { Distalis }\end{array}$ & $\begin{array}{l}0.1262 \\
0.0173\end{array}$ & $\begin{array}{l}\text { Proximalis } \\
\text { Distalis }\end{array}$ & $\begin{array}{l}0.0090 \\
0.0022\end{array}$ \\
\hline obere Extr. & $\begin{array}{l}\text { Oberarm } \\
\text { Vorderarm }\end{array}$ & $\begin{array}{l}0.2091 \\
0.0258\end{array}$ & $\begin{array}{l}\text { Oberarm } \\
\text { Vorderarm }\end{array}$ & $\begin{array}{l}0.0109 \\
0.0014\end{array}$ \\
\hline untere Fixtr. & $\begin{array}{l}\text { Oberschenkel } \\
\text { Unterschenkel }\end{array}$ & $\begin{array}{l}0.0433 \\
0.0088\end{array}$ & $\begin{array}{l}\text { Oberschenkel } \\
\text { Unterschenkel }\end{array}$ & $\begin{array}{l}0.0072 \\
0.0030\end{array}$ \\
\hline
\end{tabular}




\section{Haarbalgmuskel.}

a. Menge des Muskels in den einzelnen Körperteilen.

Tabelle VI. (Muskel).

\begin{tabular}{|c|c|c|c|c|c|}
\hline \multirow[b]{2}{*}{ Körperteile } & \multirow{2}{*}{$\begin{array}{l}\text { Volumen } \\
\text { des } \\
\text { Arrectors } \\
\text { in } 1 \mathrm{gcm}\end{array}$} & \multirow{2}{*}{$\begin{array}{l}\text { Angenom- } \\
\text { mene } \\
\text { Ausgleichs- } \\
\text { dicke } \\
\text { (rund) }\end{array}$} & \multirow{2}{*}{$\begin{array}{c}\text { Volumen } \\
\text { der } \\
\text { Muskelindiv. } \\
\text { (durchschn.) }\end{array}$} & \multicolumn{2}{|c|}{ Reihenfolge nach Volumen } \\
\hline & & & & in $1 \mathrm{qcm}$ & $\begin{array}{l}\text { Muskelindivi- } \\
\text { duum (durch- } \\
\text { schnittlich) }\end{array}$ \\
\hline Hinterhaupt & $\begin{array}{c}\mathrm{cmm} \\
1.3516\end{array}$ & $13.5 \mu$. & $\begin{array}{c}\mathrm{cmm} \\
0.0244\end{array}$ & Hinterhaupt & Untersch. \\
\hline Bauch & 0.2130 & 2.1 & 0.0211 & Rücken & Rücken \\
\hline Rücken & 1.1018 & 11.0 & 0.0637 & Vorderarm & Oberarm \\
\hline Oberarm & 0.6301 & 6.3 & 0.0542 & Oberarm & Obersch. \\
\hline Vorderarm & 0.7431 & 7.4 & 0.0428 & Untersch. & Vorderarm \\
\hline Obersch. & 0.4078 & 4.1 & 0.0432 & Obersch. & Hinterhaupt \\
\hline Untersch. & 0.4376 & 4.4 & 0.0703 & Bauch · & Bauch \\
\hline
\end{tabular}

b. Menge des Muskels in den Hauptkörperabschnitten.

Tabelle VII. (Muskel).

\begin{tabular}{|c|c|c|c|c|}
\hline \multirow{2}{*}{ Körperteile } & \multicolumn{2}{|c|}{$\begin{array}{c}\text { Volumen des Arrectors } \\
\text { in } 1 \mathrm{gcm}\end{array}$} & \multicolumn{2}{|c|}{$\begin{array}{l}\text { Volumen der Muskelindiv. } \\
\text { (durchschnittlich) }\end{array}$} \\
\hline & Köörperteile & Volumen & Körperteile & Volumen \\
\hline ganzer Körper & $\begin{array}{l}\text { Kopf } \\
\text { Rumpf } \\
\text { Extremität }\end{array}$ & $\begin{array}{l}1.3516 \mathrm{cmm} \\
0.6574 \\
0.5547\end{array}$ & $\begin{array}{l}\text { Extremität } \\
\text { Rumpf } \\
\text { Kopf }\end{array}$ & $\begin{array}{l}0.0528 \mathrm{cmm} \\
0.0424 \\
0.0244\end{array}$ \\
\hline Rumpf & $\begin{array}{l}\text { Rücken } \\
\text { Bauch }\end{array}$ & $\begin{array}{l}1.1018 \\
0.2130\end{array}$ & $\begin{array}{l}\text { Rücken } \\
\text { Bauch }\end{array}$ & $\begin{array}{l}0.0637 \\
0.0211\end{array}$ \\
\hline Extremität (a) & $\begin{array}{l}\text { obere Extr. } \\
\text { untere Extr. }\end{array}$ & $\begin{array}{l}0.6866 \\
0.4227\end{array}$ & $\begin{array}{l}\text { untere Extr. } \\
\text { obere Extr. }\end{array}$ & $\begin{array}{l}0.0570 \\
0.0480\end{array}$ \\
\hline Extremität (b) & $\begin{array}{l}\text { Distalis } \\
\text { Proximalis }\end{array}$ & $\begin{array}{l}0.5904 \\
0.5190\end{array}$ & $\begin{array}{l}\text { Distalis } \\
\text { Proximalis }\end{array}$ & $\begin{array}{l}0.0568 \\
0.0487\end{array}$ \\
\hline obere Extr. & $\begin{array}{l}\text { Vorderarm } \\
\text { Oberarm }\end{array}$ & $\begin{array}{l}0.7431 \\
0.6301\end{array}$ & $\begin{array}{l}\text { Oberarm } \\
\text { Vorderarm }\end{array}$ & $\begin{array}{l}0.0542 \\
0.0428\end{array}$ \\
\hline untere Extr. & $\begin{array}{l}\text { Unterschenkel } \\
\text { Oberschenkel }\end{array}$ & $\begin{array}{l}0.4376 \\
0.4078\end{array}$ & $\begin{array}{l}\text { Unterschenkel } \\
\text { Oberschenkel }\end{array}$ & $\begin{array}{l}0.0708 \\
0.0432\end{array}$ \\
\hline
\end{tabular}




\section{Zusammenfassung.}

Die genauen gegenseitigen Verhältnisse der Menge der Hautorgane bei dieser Rasse (Bantu-M'gonie) sind in den obigen Schlusstabellen (Tab. I-VII) angegeben, aus denen ich hier nur wichtigere Befunde kurz zusammenfassen werde, wie es von vielen Untersuchern unseres Institutes unternommen wurde.

1. Das Volumen der Schweissdrüsen auf $1 \mathrm{qcm}$ Hautflächeninhalt ist in den Tabellen (Tab. I-III) angeführt, aus denen leicht zu ersehen ist, dass das Volumen nach den Körperteilen beträchtlich verschieden ist. Bei den 7 untersuchten Körperteilen ist die Drüseumenge am Hinterhaupt $(12.8519 \mathrm{cmm}$ auf $1 \mathrm{qcm})$ am grössten, danach kommen Vorderarın (5.4770 cmm), Rücken $(4.3215 \mathrm{cmm})$, Unterschenkel (3.4535 $\mathrm{cmm})$, Oberschenkel $(3.0205 \mathrm{cmm})$ und Bauch $(2.5125 \mathrm{cmm})$; sie ist am geringsten am Oberarm $(2.4546 \mathrm{cmm})$, so dass die relative Variationsbreite der Drüsenmenge ca. 5-1 beträgt. Dieser Befund ergibt, dass das Hinterhaupt die grösste Drüsenmenge und der Oberarm die kleinste besitzt, wenngleich diesbezüglich zwischen dem Oberarm und Bauch kein merklicher Unterschied zu finden ist.

Wie die Tabelle I zeigt, schwankt die Zahl der Ausmündung der Schweissdrüsen auf $1 \mathrm{qcm}$ auch nach den Körperteilen erheblich. Die Zahl ist am Hinterhaupt (373) am grössten, damn folgen Vorderarm (213), Oberschenkel (158), Unterschenkel (158), Rücken (153) und Oberarm (14S); sie ist am geringsten am Bauch (138), so dass die relative Schwankungsbreite der Ausmündungszahl ca. 3-1 beträgt. Um diese Zahlenverhältnisse der Ausmündung gründlich zu studieren, führte ich früher ausgedehnte Untersuchungen an den verschiedenen Körperteilen, in verschiedenen Altersstufen (fetalen und postfetalen), bei beiden Geschlechtern, '/willingen und an abnorm ausgedehnter Körperoberfläche (Hydrokephalus) aus. Die Befunde dieser Untersuchungen könnten später mitgeteilt werden, worüber ich schon japanisch kurz berichtet habe.

Das Volumen der angenommenen einzelnen Sohweissdrüsen ( $\left.\frac{\text { Menge der Schweissdrüse auf } 1 \mathrm{gcm}}{\text { Zahl der Ausmündung auf } 1 \mathrm{gcm}}\right)$ ist auch nach den Körperteilen verschieden gross, ebenso bein Volumen auf $1 \mathrm{qcm}$ und bei der Ausmündungszahl, die Reihenfolge steht: Hinterhaupt $(0.0345 \mathrm{cmm})$, Rücken (0.0282 cmm), Vorderarm $(0.0257 \mathrm{cmm})$, Unterschenkel $(0.0219 \mathrm{cmm})$, Oherschenkel $(0.0191 \mathrm{cmm})$, Bauch $(0.0189 \mathrm{cmm})$ und Oberarm (0.0166 cmm); danach beträgt die relative Schwankungsbreite ca. 2-1 (Tabelle II). 
Aus dem genauen gegenseitigen Verhältnisse der Menge des Drüsenindividuums, der gesamten Drüsenmenge und Ausmündungszahl auf einem bestimmten Hautflächeninhalt kann man leicht erkennen,-wie schon von vielen Mitarbeitern unseres Institutes beobachtet-dass das Volumen der Schweissdrüse und die Zahl der Drüsenausmündung auf $1 \mathrm{gcm}$ sowie das Volumen des Drüsenindividuums im grossen und ganzen miteinander parallel gehen; nach den Körperteilen ist eine kleine Schwankung erkenubar.

Die relative Schwankungsbreite der Drüsenmenge auf $1 \mathrm{qcm}$ ist am grössten (1-5), danach kommt die der Ausmündungszahl (3-1) und zuletzt die des Drüsenindividuums (2-1). Es ist beachtenswert, dass das Volumen des Drüsenindividuums nach den Körperteilen keine so erhebliche individuelle Schwankung zeigt wie die der gesamten Drüsenmenge auf einen bestimmten Hautflächeninhalt.

Die Tabelle III zeigt uns, dass das durchschnittliche Volumen der Schweissdrüse unter den drei eingeteilten Hauptkörperteilen-Kopf, Rumpf und Extremitäten-im Kopf $(12.8519 \mathrm{cmm})$ am grössten, in den Extremitäten $(3.6013 \mathrm{cmm})$ und im Rumpf $(3.4124 \mathrm{cmm})$ fast gleich gross ist. Beim Rumpf ist die Menge der Schweissdrüse im Rücken $(4.3125 \mathrm{cmm})$ ca. 2 mal grösser als am Bauch $(2.5123 \mathrm{cmm})$. Im allgemeinen ist die Drüsenmenge auf der dorsalen Körperseite grösser als auf der ventralen. Diese auffallende Tatsache ist schon von unseren Mitarbeitern an verschiedenen Individuen beobachtet worden. Es wäre sehr interessant, diese Befunde entwicklungsgeschichtlich und vergleichend-anatomisch weiter zu studieren. Die Zahl der Ausmündung verhält sich hier umgekehrt; so ist das Volumen des Drüsenindividuums im Rücken (0.0282 $\mathrm{cmm})$ auffallend grösser als am Bauch $(0.0189 \mathrm{cmm})$.

Bei den Extremitäten ist die Drüsenmenge auf $1 \mathrm{qcm}$, die des einzelnen Drüsenindividuums und die Zahl der Ausmündung an der oberen Extremität viel grösser als an der unteren. Die obere Extremität hat am Vorderarm eine viel grössere Drüsenmenge und Ausmündungszahl als am Oberarm und die untere Extremität am Unterschenkel als am Oberschenkel. Es lässt sich also sagen, dass bei den Extremitäten die Drüsenmenge auf $1 \mathrm{qcm}$, das Volumen des Drüsenindividuums und die Ausmündungszahl in den distalen Regionen viel grösser als in den proximalen sind. Diese beachtenswerte Tatsache ist auch von vielen Untersuchern öfters beobachtet worden.

2. Das Volumen der Talgdrüsen aưf $1 \mathrm{qcm}$ Hautflächeninhalt (Tab. IV und V) ist, wie bei der Schweissdrüse, im Hinterhaupt (2.6542 $\mathrm{cmm}$ ) am grössten, dann kommen Rücken $(0.3803 \mathrm{cmm})$, Oberarm 
(0.2091 cmin), Bauch $(0.0521 \mathrm{cmm})$, Oberschenkel $(0.0433 \mathrm{cmm})$, Vonderarm $(0.0258 \mathrm{cmm})$ und zuletzt Unterschenkel $(0.0088 \mathrm{cmm})$, so dass die relative Schwankungsbreite auffallend gross, ca. 300-1 ist. Es lässt sich also sagen, dass die relative Schwankungsbreite der Menge der Talgdrüse bedeutend grösser als die der Schweissdrüse ist. Dieser Befund stimmt auch mit den Befunden von anderen Untersuchern überein, weungleich die Grösse der relativen Schwankungsbreite der beiden Drüsenarten (Talgund Schweissdrüsen) nach den Individuen beträchtlich verschieden ist.

Die durchschnittliche Menge der Talgdrüse bei einer Haargruppe, beliebig je vorı den 10 Haargruppen der einzelnen Körperteile ausgewählt, ist im Hinterhaupt $(0.0369 \mathrm{cmm})$ am grössten, dann folgen Rücken $(0.0166 \mathrm{cmm})$, Oberarm $(0.0109 \mathrm{cmm})$, Oberschenkel $(0.0072 \mathrm{cmm})$, Bauch $(0.0047 \mathrm{cmm})$, Unterschenkel $(0.0030 \mathrm{cmm})$ und zuletzt Vorderarm $(0.0014$ cmm), so dass die relative Schwankungsbreite ca. 26-1 ist. Demzufolge kann man wohl sagen, dass das durchschnittliche Volumen der Talgdrüsen bei einer Haargruppe und die gesamte Drüsenmenge auf $1 \mathrm{qcm}$ im Gegensatz zur Schweissdrüse so beträchtliche Schwankung nach dẹ Körperteilen zeigen.

Bei den 7 untersuchten Körperteilen konnte ich die Haargruppe ohne Begleitung der Talgdrüse am Bauch, Unterschenkel, Ober- und Vorderarm beobachten. Diese talgdrüsenlose Haargruppe kommt oft im allgemeinen auf den Körperteilen mit geringer Talgdrüsenmenge auf einen bestimınten Hautflächeninhalt vor.

In den drei Hauptabteilungen des Körpers fand ich, dass die Menge der Talgdrüse auf $1 \mathrm{gcm}$ im Kopf am grössten ist, dann folgt der Rumpf, an geringsten ist sie in den Extremitäten. Beim Rumpf ist sie im Rücken über 6 mal grösser als am Bauch; also auf der Dorsalseite grösser als auf der Ventralseite. Bei den Extremitäten ist sie an der oberen Extremität grösser als an der unteren, bei der oberen Extremität am Oberarn etwas grösser als am Vorderarm und bei der unteren Extremität am Oberschenkel mehr als am Unterschenkel. Man kamn also wohl sagen, dass bei den Extremitäten das Volumen der Talgdrüse auf $1 \mathrm{gcm}$ in deu proximalen Regionen grösser als in den distalen ist; diese Verhältnisse finden sich bei der Schweissdrüse ganz umgekehrt.

Die durchschnittliche Menge der Talgdrüse bei einer Haargruppe ist im Kopf am grössten, dann folgt der Rumpf und zuletzt die Extremitäten. Beim Rumpf ist sie im Rücken ca. 4 mal grösser als am Bauch. Bei den Extremitäten ist sie an der oberen Extremität etwas reichlicher als an der unteren, und proximal grösser als distal. In der oberen Extremität ist die Menge am Oberarm grösser als am Vorderarm und in der unteren 
am Oberschenkel grösser als am Unterschenkel. Aus obigen Befunden ergibt sich, dass die durchschnittliche Menge der Talgdrüse bei einer Haargruppe und die Menge der Talgdrüsen auf einen bestimmten Hautflächeninhalt im grossen und ganzen parallel stehen, und dass sie nach den Körperteilen einer grossen Schwankung unterworfen sind.

3. Die Menge der Haarbalgmuskel $\mathrm{n}$ auf $1 \mathrm{qcm}$ Hautflächeninhalt (Tab. VI und VII) ist am Hinterhaupt $(1.3516 \mathrm{cmm})$ am grössten, danach kommen Rücken $(1.1018 \mathrm{cmm})$, Vorderarm $(0.7431 \mathrm{cmm})$, Oberarm $(0.6301$ cmm), Unterschenkel $(0.4376 \mathrm{cmm})$, Oberschenkel $(0.4078 \mathrm{cmm})$ und zuletzt Bauch $(0.2130 \mathrm{cmm})$, so dass die relative Schwankungsbreite sich auf ca. 6-1 beläuft.

Bei den 3 Hauptkörperteilen ist die Menge auf $1 \mathrm{gcm}$ im Kopf $(1.3516 \mathrm{cmm})$ am grössten, dann kommt der Rumpf $(0.6574 \mathrm{cmm})$ und zuletzt Extremitäten $(0.5547 \mathrm{cmm})$. Beim Rumpf ist die Menge, wie bei der Talg- und Schweissdrüse, im Rücken $(1.1018 \mathrm{cmm})$ ca. 5 mal grösser als am Bauch $(0.2130 \mathrm{cmm})$. Bei den Extremitäten ist sie an der oberen Extremität $(0.6899 \mathrm{cmm})$ grösser als an der unteren $(0.4227 \mathrm{cmm})$. Die obere Extremität besitzt am Vorderarm $(0.07431 \mathrm{cmm})$ eine viel grössere Drüsenmenge als am Oberarı $(0.6301 \mathrm{cmm})$ und die untere Extremität am Unterschenkel $(0.4375 \mathrm{cmm})$ eine grössere als am Oberschenkel $(0.4078 \mathrm{cmm})$. Also kann gesagt werden, dass bei den Extremitäten die Menge der Haarbalgmuskeln auf $1 \mathrm{qcm}$ in den distalen Regionen viel reichlicher als in den proximalen zu finden ist. Diese Verhältnisse des Haarbalgmuskels bei den Extremitäten stimmen fast mit den der Schweissdrüsen überein, dagegen bei den Talgdrüsen ganz umgekehrt.

Die durchschnittliche Menge der Muskeln bei einer Haargruppe ist im Unterschenkel $(0.0703 \mathrm{cmm})$ am grössten, dann folgen der Rücken $(0.0637 \mathrm{cmm})$, Oberarm $(0.0542 \mathrm{cmm})$, Oberschenkel $(0.0432 \mathrm{cmm})$, Vorderarm $(0.0428 \mathrm{cmm})$, und zuletzt Hinterhaupt $(0.0244 \mathrm{cmm})$, so dass die relative Schwankungsbreite ca. 1-3 beträgt. Es ist beachtenswert, dass der Unterschenkel die grösste durchschnittliche Menge der Muskeln bei einer Haargruppe besitzt.

Bei den Hauptkörperabteilungen ist die durchschnittliche Menge der Muskeln bei einer Haargruppe in den Extremitäten $(0.0528 \mathrm{cmm}) \mathrm{am}$ grössten, dann folgt der Rumpf $(0.0424 \mathrm{cmm})$ und zuletzt der Kopf $(0.0244 \mathrm{cmm})$. Beim Rumpf ist sie im Rücken $(0.0637 \mathrm{cmm}) 3 \mathrm{mal}$ grösser als am Bauch $(0.0211 \mathrm{cmm})$. Und sie ist bei den Extremitäten an der unteren $(0.0570 \mathrm{cmm})$ ein wenig grösser als an der oberen $(0.0480$ $\mathrm{cmm})$ und bei beiden distal $(0.0566 \mathrm{cmm})$ grösser als proximal $(0.0487$ cmm). Bei der oberen Extremität ist die Menge am Oberarm (0.0542 
$\mathrm{cmm}$ ) grösser als am Vorderarm (0.0428 cmm). Bei der uuteren iat sie am Unterschenkel $(0.0708 \mathrm{cmm})$ reichlicher als am Oberschenkel $(0.0432$ cmm).

Zum Schluss spreche ich Herrn Professor K. Okajima für seino stete liebenswürdige Unterstützung bei dieser Arbeit meinen herzlichșten Dank aus.

\section{Literatur.}

1) Okajima, K. u. Kanaizuka, Z., Quantitative Untersuchung des Haarbalgmuskels bei den Säugetieren. Folia Anat. Japon. Bd. VII. 1929.

2) Taniguchi, T., Quantitative Untersuchung der Anhangsorgane der Haut hei dem japanischen Neugeborenen. Folia Anat. Japon. Bd. X. 1931.

3) Koibuchi, S., Quantitative Untersuchung der Anhangsorgane der Haut bei dem japanischen Kind. Folia Anat. Japon. Bd. X. 1932.

4) Yamada, K., Quantitative Untersuchung der Anhangsorgane der Haut bei dem Deutschen. Folia Anat. Japon. Bd. X. 1932.

5) Kosaka, Y., Quantitative Untersuchung der Anhangsorgane der Haut bei einem japanischen Fetus. Folia Anat. Japon. Bd. X. 1932.

6) Taniguchi, T., Kosaka, Y. und Nakano, T., Quantitative Untersuchung der Anhangsorgane der Haut bei einem koreanischen Kind. Folia Anat. Japon. Bd. XI. 1933.

7) Kawai, M., Quantitative Untersuchung der Anhangsorgane der Haut bei einem erwachsenen Ainu. Folia Anat. Japon. Bd. XI. 1933.

8) Kawai, M., Quantitative Untersuchung der Anhangsorgane der Haut bei einem japanischen Mädchen. Folia Anat. Japon. Bd. XI. 1933.

9) Kawaji, T., Quantitative Untersuchung der Anhangsorgane der Haut bei den Finnen. Folia Anat. Japon. Bd. XII. 1934.

10) Kawaji, T., Quantitative Untersuchung der Anhangsorgane der Haut des Handrückens bei den Japanern. Folia Anat. Japon. Bd. XII. 1934.

11) Ito, T., Quantitative Untersuchung der Anhangsorgane der Haut bei einer japanischen Frau. Folia Anat. Japon. Bd. XII. 1934.

12) Taniguchi, T., Quantitative Untersuchung der Anhangsorgane der Haut bei einem Hydrokephalus. Folia Anat. Japon. Bd. XIII. 1935.

13) Sh i ba y a ma, H., Quantitative Untersuchung der Anhangaorgane der Haut bei dem Bantu-Xosa. Folia Anat. Japon. Bd. XIII. 1935.

14) Taniguchi, T., Utber die Verteilung der Schweissdrüsen beim Menschen. Nagoya Iji Shinshi. Bd. 8. 1934 (Japanisch). 